\title{
Study of Instability of Streaming Rivlin-Ericksen Fluid in Porous Medium
}

\author{
Bappaditya Jana, Joyanta Sarkar
}

\begin{abstract}
There are many elastoviscous fluids that can be characterised neither by Maxwell's constitutive relations nor by Oldroyd's constitutive relations. One such class of viscoelastic fluids is the Rivlin-Ericksen's fluid. RIVLIN and ERICKSEN] have proposed a theoretical model for such viscoelastic fluid. The behaviour of surface waves propagating between two Rivlin-Ericksen elastico-viscous fluids is examined. The investigation is made in the presence of a vertical electric field and a relative horizontal constant velocity. The influence of both surface tension and gravity force is taken into account. Due to the inclusion of streaming flow a mathematical simplification is considered. The viscoelastic contribution is demonstrated in the boundary conditions. From this point of view the approximation equations of motion are solved in the absence of viscoelastic effects. The solutions of the linearized equations of motion under nonlinear boundary conditions lead to derivation of a nonlinear equation governing the interfacial displacement and having damping terms with complex coefficients.

Index terms- viscoelastic effects; perturbation; permeability; elastoviscous fluids; porous medium; thermosolutal instability; thermohaline convection; convecting layers hydrothermal circulation; oscillatory modes; non-Newtonian fluids
\end{abstract}

\section{Introduction}

Now a days a class of polymers are used for manufacturing parts of space-crafts, aeroplanes, tyres, belt conveyers, ropes, cushions, seats, foams, plastics, engineering equipments, etc. Recently, polymers are also used in agriculture, communication appliances and in biomedical applications. When fluid permeated a porous material, the gross effect is When fluid permeated a porous material, the gross effect is represented by Darcy's law. As a result of this macroscopic law, the usual viscous terms in the equations of Rivlin-Ericksen's elastoviscous fluid motion is replaced with

$$
\left[-\frac{1}{k_{1}}\left(\mu+\mu^{\prime} \frac{\partial \vec{q}}{\partial t}\right)\right],
$$

where $\mu$ and $\mu^{\prime}$ are the viscosity and viscoelasticity in the thermal instability of the Rivlin-Ericksen fluid, k1 is the medium permeability and $\mathrm{q} \square$ is the Darcian (filter) velocity of the fluid. STOMMEL and FEDOROR [6] and LINDEN [7] have remarked that the length-scale characteristics of double diffusive convecting layers in the ocean may be sufficiently large to make the Earth rotation important to their formation. Moreover, the rotation of the Earth distorts the boundaries of a hexagonal convection cell in a fluid through a porous medium and the distortion plays an important role in the extraction of energy in the geothermal regions. The problem of thermal instability in fluid in porous mediums is of importance in geophysics, soil sciences, ground water hydrology and astrophysics. The scientific importance of the field has also increased because hydrothermal circulation is the dominant heat transfer mechanism in the development of young oceanic crust (LISTER [8]). The stability criteria are discussed theoretically and illustrated graphically in which stability diagrams are obtained. Regions of stability and instability are identified for the electric fields versus the wavenumber for the wavetrain of the disturbance. Numerical calculations showed that the ratio of the dielectric constant plays a dual role in the stability criteria. The damping role for the viscosity coefficient is observed. The viscoelasticity coefficient plays two different roles. A stabilizing influence is observed through the linear scope and a destabilizing role in the nonlinear stability picture is seen.

Keeping in mind the importance of ground water hydrology, soil sciences, geophysics and astrophysics, the thermal instability of the Rivlin-Ericksen elasto-viscous rotating fluid that permeates with suspended particles under variable gravity field in porous medium has been considered in this topic.

\section{Instability Of Superposed Streaming Fluids Through A Porous Medium:-}

This chapter treats the Kelvin-Helmholtz instability arising at the interface separating 2 superposed, viscous, electrically conducting fluids through a porous medium in the presence of a uniform $2 \mathrm{D}$ horizontal magnetic field. The stability motion was also assumed to be uniform, 2D, and horizontal. By applying the normal mode technique to the linearized perturbation equations, the dispersion relation was derived. The stability analysis was carried out for fluids of high kinematic viscosities. It was found that both viscosity and porosity suppressed the 
stability, while streaming motion had a destabilizing influence. The Kelvin-Helmholtz discontinuity arising at the plane interface between 2 superposed streaming fluids is of prime importance in various astrophysical, geophysical, and laboratory situations.

\section{Kelvin-Helmholtz Instability Of Rivlin-Ericksen Viscoelastic Fluid In Porous Medium:-}

Kelvin-Helmholtz instability of Rivlin-Ericksen elasticoviscous fluid in porous medium is considered. The case of two uniform streaming fluids separated by a horizontal boundary is considered. It is found that for the special case when perturbations in the direction of streaming are ignored, perturbation transverse to the direction of streaming are found to be unnafected by the presence of streaming. In every other direction, a minimum value of wave-number has been found and the system is unstable for all wave-numbers greater than this minimum wave number.

When two superposed fluids flow one over the other with a relative horizontal velocity, the instability of the plane interface between the two fluids, when it occurs in this instance, is known as 'Kelvin-Helmholtz instability'. The instability of the plane interface separating two uniform superposed streaming fluids, under varying assumptions of hydrodynamics, has been discussed in the celebrated monograph by Chandrasekhar [1]. The experimental observation of the Kelvin-Helmholtz instability has been given by Francis [2]. The medium has been assumed to be non-porous. With the growing importance of viscoelastic fluids in modern technology and industries and the investigations on such fluids are desirable. The Rivlin-Ericksen fluid is one such viscoelastic fluid. Many research workers have paid their attention towards the study of Rivlin-Ericksen fluid. Johri [3] has discussed the viscoelastic Rivlin-Ericksen incompressible fluid under time-dependent pressure gradient. Sisodia and Gupta [4] and Srivastava and Singh [5] have studied the unsteady flow of a dusty elasticoviscous Rivlin-Ericksen fluid through channel of different cross-sections in the present of the time dependent pressure gradient. Recently, Sharma and Kumar [6] have studied the thermal instability of a layer of RivlinEricksen elastico-viscous fluid acted on by a uniform rotation and found that rotation has a stabilizing effect and introduces oscillatory modes in the system.

The flow through a porous medium has been of considerable interest in recent years particularly among geophysical fluid dynamicists. An example in the geophysical context is the recovery of crude oil from the pores of reservoir rocks. A great number of applications in geophysics may be found in a recent book by Phillips [7]. The gross effect when the fluid slowly percolates through the pores of the rock is given by Darcy's law. As a result, the usual viscous term in the equation of motion of Rivlin-Ericksen fluid is replaced by the resistance term

$$
\left[-\frac{1}{k_{1}}\left(\mu+\mu^{\prime} \frac{\partial}{\partial t}\right) \vec{q}\right],
$$

Where $\mu$ and $\mu 0$ are the viscosity and viscoelasticity of the Rivlin-Ericksen fluid, $\mathrm{k} 1$ is the medium permeability and is the Darcian (filter) velocity of the fluid. Generally, it is accepted that comets consists of a dusty 'snowball' of a mixture of frozen gases which, in the process of their journey, changes from solid to gas and vice-versa. The physical properties of comets, meteorites and interplanetary dust strongly suggest the importance of porosity in astrophysical contex (McDonnel [8]). The instability of the plane interface between two uniform superposed and streaming fluids through porous medium has been investigated by Sharma and Spanos [9]. More recently, Sharma et al. [10] have studied the thermosolutal convection in Rivlin-Ericksen fluid in porous medium in the presence of uniform vertical magnetic field. Keeping in mind the importance of non-Newtonian fluids in modern technology and industries and various applications mentioned above, KelvinHelmholtz instability of Rivlin-Ericksen viscoelastic fluid in porous medium has been considered in the present topic..

\section{On Rivlin-Ericksen Elasto-Viscous Fluid Heated And Soluted \\ From Below In The Presence Of Compressibility,Rotation And Hall Current-}

A layer of compressible, rotating, elastico-viscous fluid heated \& soluted from below is considered in the presence of vertical magnetic field to include the effect of Hall currents. Dispersion relation governing the effect of viscoelasticity, salinity gradient, rotation, magnetic field and Hall currents is derived. For the case of stationary convection, the Rivlin-Erickson fluid behaves like an ordinary Newtonian fluid. The compressibility, stable solute gradient, rotation and magnetic field postpone the onset of thermosolutal instability whereas Hall currents are found to hasten the onset of thermosolutal instability in the absence of rotation. In the presence of rotation, Hall currents postpone/hasten the onset of instability depending upon the value of wavenumbers. Again, the dispersion relation is analyzed numerically \& the results depicted graphically. 
The stable solute gradient and magnetic field (and corresponding Hall currents) introduce oscillatory modes in the system which were non-existent in their absence. The case of overstability is discussed \& sufficient conditions for non-existence of overstability are derived. For thermosolutal convection, buoyancy forces can arise not only from density differences due to variation in temperature gradient, but also from those due to variation in solute concentration and this double diffusive phenomenon has been extensively studied in recent years due to its direct relevance in the field of chemical engineering, astrophysics, and oceanography. Veronis [20] studied the problem of thermohaline convection in the layer of fluid heated from below and subjected to a stable salinity gradient. The physics is quite similar to Veronis thermohaline configuration in the stellar case, in that helium acts like salt raising the density and in diffusing more slowly than heat. The heat and solute being two diffusing components, thermosolutal (double-diffusive) convection is the general term dealing with such phenomenon.

The Hall current is likely to be important in many geophysical and astrophysical situations as well as in flows of laboratory plasmas. Sherman and Sutton [17] have considered the effect of Hall currents on the efficiency of a magneto-fluid dynamic (MHD) generator. The effect of Hall currents on the thermal instability of electrically conducting fluid in the presence of a uniform vertical magnetic field has been studied by Gupta [5]. Sharma and Gupta [14] investigated the effect of Hall currents on thermosolutal instability of a rotating plasma.

When the fluids are compressible, the equations governing the system become quite complicated. Spiegal and Veronis [18] have simplified the set of equations governing the flow of compressible fluids under the assumption that the depth of the fluid layer is much smaller than the scale height as defined by them, if motions of infinitesimal amplitude are considered. Sharma and Gupta [16] have considered the effect of suspended particles and Hall currents on the stability of compressible fluids saturating a porous medium. Chandrasekhar [3] has given a detailed account of the theoretical and experimental results on the onset of thermal instability (B'enard convection) in an incompressible, viscous Newtonian fluid layer under varying assumptions of hydrodynamics and hydromagnetics. In all these studies, fluid has been considered to be Newtonian. In case of non-Newtonian fluids, Bhatia and Steiner[2] have studied the problem of thermal instability of a Maxwellian viscoelastic fluid in the presence of rotation and found that rotation has a destabilizing influence in contrast to the stabilizing effect on a viscous Newtonian fluid.

Recently, Halder [7] investigated the flow of blood through a constricted artery in the presence of an external transverse magnetic field using Adomian's decomposition method. The expressions for two term approximation to the solution of stream function, axial velocity component and wall shear stress are obtained in this analysis. In another application, Ajadi [1] studied the isothermal flow of a dusty viscous incompressible conducting fluid between two types of boundary motions-oscillatory and non-oscillatory under the influence of gravitational force. There is growing importance of non-Newtonian viscoelastic fluids in chemical technology, industry and geophysical fluid dynamics. Sharma and Kumar [15] have studied the effect of rotation on thermal instability in Rivlin-Erickson elastico-viscous fluids. Recently, Sunil et. al. [19] have studied the effect of Hall currents on thermosolutal instability of compressible Rivlin-Erickson fluids. Keeping in mind the conflicting tendencies of magnetic field and rotation

while acting together and the growing importance of non-Newtonian fluids in modern technology, industry, chemical technology and dynamics of geophysical fluids; we are motivated to study the thermosolutal instability of a compressible Rivlin-Erickson fluid in the presence of rotation and Hall currents. This problem to the best of our knowledge, has not been investigated yet.

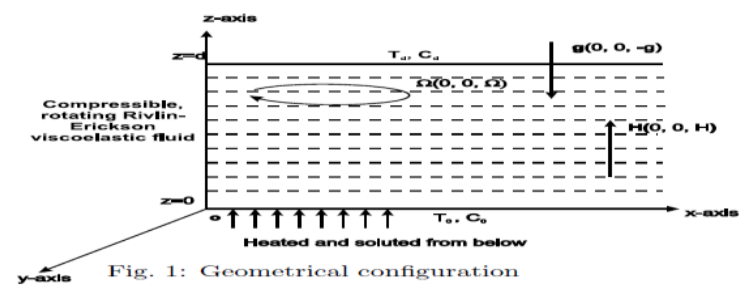

\section{The Instability Of Streaming Walter's Fluid In Porous Medium In Hydromagnetics-}

The instability of the plane interface separating the two uniform, superposed, electrically conducting and counter - streaming elastico-viscous fluids through a porous medium is examined for viscoelastic polymeric solutions in the presence of a horizontal magnetic field and also in the presence and absence of surface tension. These solutions are known as Walters' (modelB $\square \square$ ) fluids and thi rheology is approximated by the Walters' (model B $\square \square$ ) constitutive relations, proposed by Walters (1962). In the absence of surface tension, the perturbations transverse to the direction of streaming are found to be unaffected by the presence of streaming if 
perturbations in the direction of streaming are ignored, whereas for perturbations in all other directions, there exists the instability for a certain wave number range. The magnetic field and surface tension are able to suppress this Kelvin-Helmoltz instability for small wave length perturbations, and the medium porosity and the viscoelasticity reduce the stability range given in terms of a difference in streaming velocities and the Alfven velocity.

The instability of the plane interface separating the two superposed semi - infinite fluids flowing with different velocities has been considered by Helmholtz (1868) and Kelvin (1910) and a review of this Kelvin Helmholtz instability, under varying assumptions of hydrodynamics and hydromagnetics, has been given by Chandrashekhar (1961). Helmholtz stated that every perfect geometrically sharp edge by which a fluid flows out tear it as under and establish a surface of separation; however, slowly the rest of the fluid may more. A good review of the interface between two fluids in relative motion has been given by Gerwin (1968). Alterman (1961) has studied the effect of surface tension to the Kelvin-Helmholtz instability of two rotating fluids. Reid (1961) studied the effect of surface tension and viscosity on the stability of two superposed fluids. Bellman and Pennington (1954) further investigated in detail illustrating the combined effects of viscosity and surfacetension. The medium has been assumed to be non - porous in the above studies.

\section{VI. .Almost Undirectional Flows -}

In this chapter, two different classes of flows are examined in the limit of almost rectilinear flow domains, by using perturbation analysis of the full Navier-Stokes equations. These are:

(a) Lubrication flows: these are confined or free surface flows with parabolic velocity profiles, under almost rectilinear boundaries or free surfaces. Typical examples are flow in converging and diverging channels, flow in pipes, and flow of thin films on substrates.

(b) Stretching flows: these are free surface flows of plug-like velocity profile under almost rectilinear free surfaces, such as jet flows.

Prototypes of these flows, such as flows in non-rectilinear domains, development of wet films under surface tension, and spinning/casting/blowing of polymeric fibers/sheets/films, are depicted..

VII. Properties Of Continuum Fluid:-

Macroscopic and Microscopic Balances: The control volume is an arbitrary synthetic cut in space which can be either fixed or moving. It is appropriately chosen within or around the system under consideration, in order to apply the laws that describe its behavior. In flow systems, these laws are the equations of conservation (or change) of mass, momentum, and energy. To obtain information on average or boundaryquantities (e.g., of the velocity and the temperature fields inside the flow system), without a detailed analysis of the flow, the control volume is usually taken to contain or to coincide with the real flow system. The application of the principles of conservation to this finite system produces the macroscopic conservation equations.

However, in order to derive the equations that yield detailed distributions of fields of interest, the control volume must be of infinitesimal dimensions that can shrink to zero, yielding a point-volume. This approach reduces the quantities to point-variables. The application of the conservation principles to this infinitesimal system produces the microscopic or differential conservation equations. In this case, there is generally no contact between the imaginary boundaries of the control volume and the real boundaries of the system. It is always convenient to choose the shape of the infinitesimal control volume to be similar to that of the geometry of the actual system; a cube for a rectangular geometry, an annulus for a cylindrical geometry and a spherical shell for a spherical geometry.

\section{Laminar Boundary Layer Flows:-}

- Boundary Layer Flow: In this chapter, we consider flows near solid surfaces known as boundary layer flows. One way of describing these flows is in terms of vorticity dynamics, i.e., generation, diffusion, convection, and intensification of vorticity. The presence of vorticity distinguishes boundary layer flows from potential flows, which are free of vorticity. In two-dimensional flow along the xy-plane, the vorticity is given by

$$
\boldsymbol{\omega} \equiv \nabla \times \mathbf{u}=\left(\frac{\partial u_{y}}{\partial x}-\frac{\partial u_{x}}{\partial y}\right) \mathbf{e}_{k},
$$

and is a measure of rotation in the fluid. As discussed in Chapter 6, vorticity is generated at solid boundaries. 


\section{Equations And Figures :-}

9.1 Lubrication Flows: Lubrication flows are most applicable to processing of materials in liquid form, such as polymers, metals, composites and others. One-dimensional approximations can be derived from simplified mass and momentum balances by means of control volume principles, or by simplifying the general equations of change. This leads to the celebrated Reynolds equation [1],

$$
F(h, p, S t, C a)=0 \text {, }
$$

where $h(x)$ is the thickness of the narrow channel or of the thin film, p is the pressure, St is the Stokes number,

$$
S t \equiv \rho g L^{2} / \eta V \text {, and Ca is the capillary number, }
$$

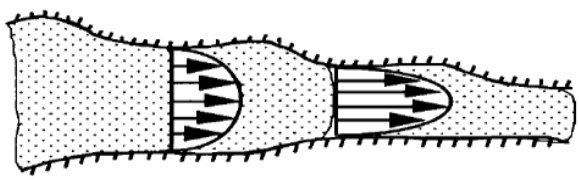

converging channel flow

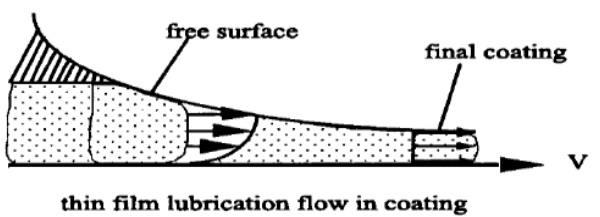

thin film lubrication flow in coating

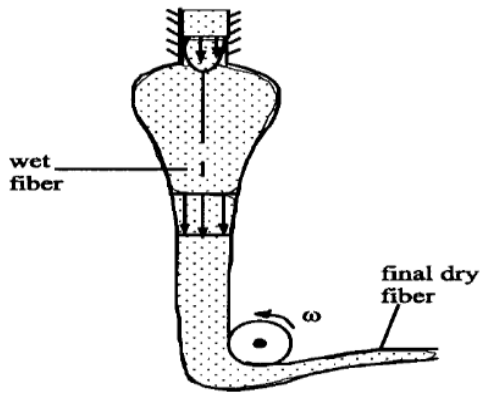

stretching flow in fiber spinning

Figure 9.1. Confined and film lubrication flows and stretching flows.

$$
C a \equiv \eta V / \sigma \text {, }
$$

that appears due to surface tension along an interface. Equation (9.1) can be solved:

(a) for the pressure distribution and other relevant quantities, such as load capacity, friction, cavitation etc., when the thickness $\mathrm{h}(\mathrm{x})$ is known. Typical applications are lubrication of solid surfaces in relative motion, such as journalbearing, piston-cylinder and piston-rings of engines [2].

(b) for the thickness $\mathrm{h}(\mathrm{x})$, when the pressure is known. Typical examples are formation of thin films and coating applications [3].

9.1.1 Lubrication vs. Rectilinear Flow: The lubrication approximation for flows in nearly rectilinear channels or pipes, with nearly parallel walls can be derived intuitively from the complete set of flow equations. Mass conservation requires constant flow rate:

$$
\frac{\partial u_{x}}{\partial x}=0, \quad u_{z}=0, \quad u_{x}=f(z) .
$$

Conservation of linear momentum in the flow direction requires pressure and viscous force balance in the same direction:

$$
\frac{\partial p}{\partial x}=\eta \frac{\partial u_{x}^{2}}{\partial z^{2}} \text {. }
$$

$\partial x$ The pressure along the channel, equal to $\Delta \mathrm{p} / \Delta \mathrm{L}$, where $\Delta \mathrm{p}$ is the pressure difference over a distance $\Delta \mathrm{L}$, Fig. 9.2 . For constant pressure gradient, the momentum equation predicts linear shear stress and parabolic velocity profile. In these problems, the mechanism of fluid motion is simple; material flows from regions of high pressure to regions of low pressure (Poiseuille-type flow). 


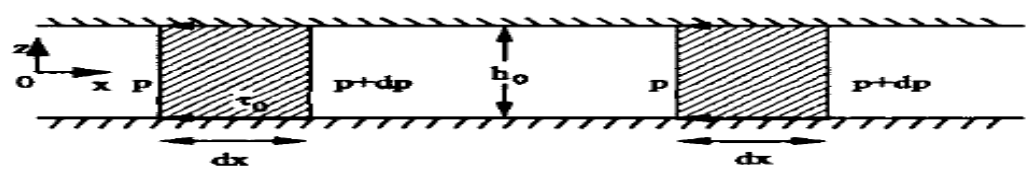

(a)

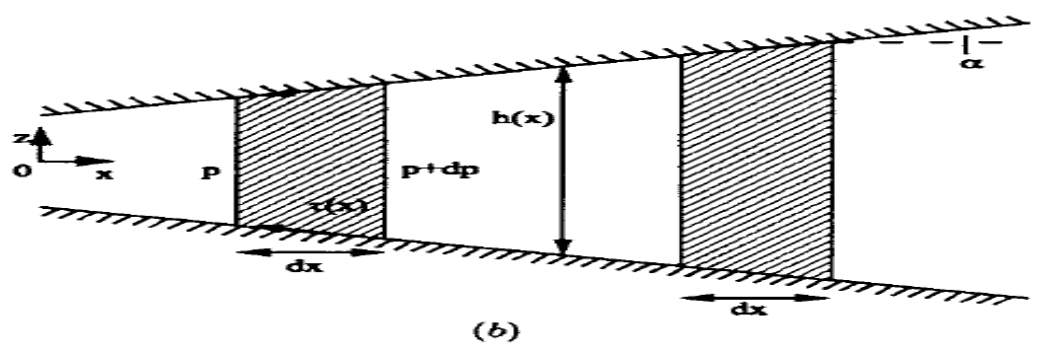

Figure 9.2. Force balance in (a) rectilinear flow, h0dp $=2 \tau d x$, and (b) lubrication flow, $h(x) d p(x)=2 \tau(x) d x$. When one or both walls are at a slight inclination $\alpha$ relative to each other, the same governing equations are expected to hold. Now, however, they may locally be weak functions of $\mathrm{x}$ of order $\alpha$. Take for instance, the pressure gradient in lubrication applications where the flow may be accelerating or decelerating, in converging or diverging channels, respectively. In such cases, $\partial \mathrm{p} / \partial \mathrm{x}$ is not constant along the channel. This can be seen in Fig. 9.2(b) where the pressure force needed to move two cones of liquid of the same width, dx, at two different positions along the channel isdifferent. Consequently, both $\partial \mathrm{p} / \partial \mathrm{x}$ and the velocity are functions of $\mathrm{x}$. Therefore, we have

$$
\begin{aligned}
& \frac{\partial u_{x}}{\partial x}+\frac{\partial u_{z}}{\partial z}=0 \\
& \text { and } \frac{\partial p(x)}{\partial x}=\eta \frac{\partial^{2} u_{x}}{\partial z^{2}} .
\end{aligned}
$$
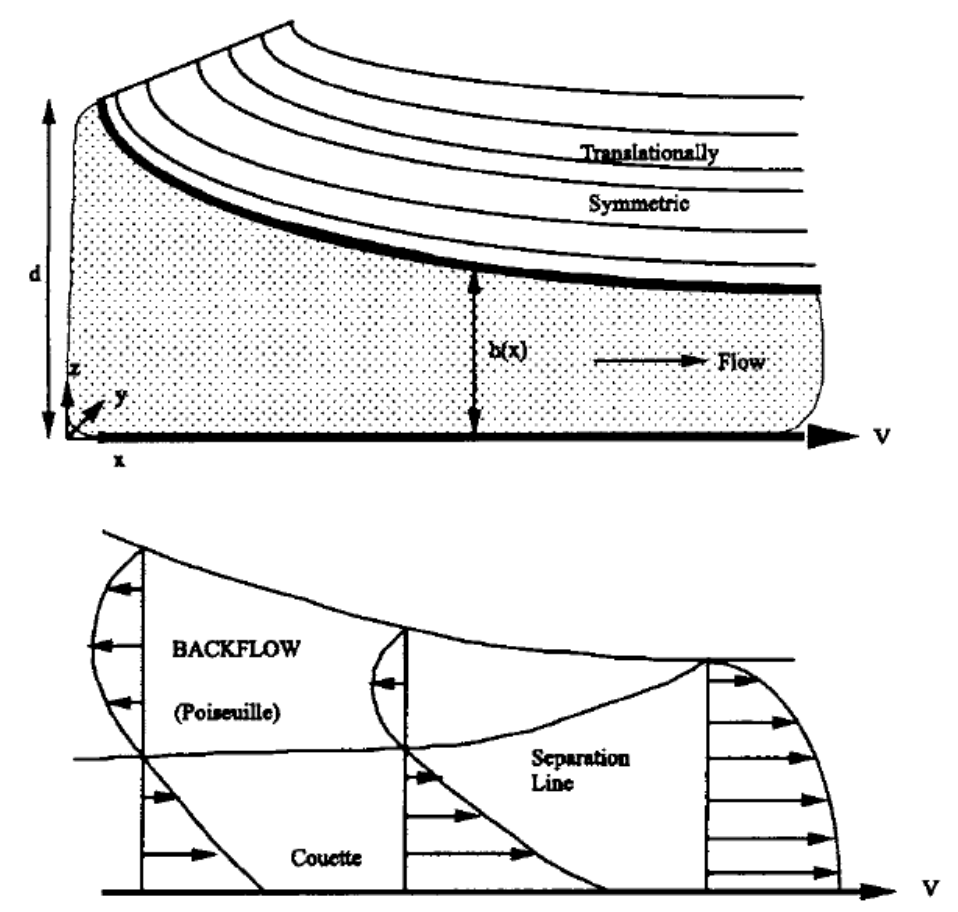

Figure 9.3. Geometry of one-dimensional lubrication flow. The velocity profiles along the channel are a mixture of Couette and Poiseuille flow. 
Equations (9.3) and (9.5) express conservation of linear momentum for a control volume. They both indicate that, due to negligible convection, there is no accumulation of momentum. Consequently, the forces capable of producing momentum are in equilibrium.

As shown in Fig. 9.2, the forces on a control volume of width $\mathrm{dx}$, are the net pressure force (dp/dx)A(x) and the shear stress force $2 \tau x y d x$. However, the underlying mechanism in lubrication flows may be more complex than in Poiseuille flow. Consider, for instance, the schematic in Fig. 9.3. Through the action of viscous shear forces, the moving wall on one side sweeps fluid into a narrowing passage. This gives rise to a local velocity profile of Couette-type, $u x=V y / h$, with flow rate, $Q=V h / 2$. Since $Q$ is constant, in order to conserve mass, $h(x)$ is decreasing. The flow then sets up a pressure gradient, in order to supply a Poiseuille-type flow component that redistributes the fluid and maintains a constant flow rate.

9.1.2 Derivation of Lubrication Equations: The lubrication equations can be alternatively derived by



$$
\begin{aligned}
\frac{\partial u_{x}}{\partial x}+\frac{\partial u_{z}}{\partial z}=0, & \\
\rho\left(\frac{\partial u_{x}}{\partial t}+u_{x} \frac{\partial u_{x}}{\partial x}+u_{z} \frac{\partial u_{x}}{\partial z}\right) & =-\frac{\partial p}{\partial x}+\eta\left[\frac{\partial u_{x}^{2}}{\partial x^{2}}+\frac{\partial u_{x}^{2}}{\partial z^{2}}\right], \\
\rho\left(\frac{\partial u_{z}}{\partial t}+u_{x} \frac{\partial u_{z}}{\partial x}+u_{z} \frac{\partial u_{z}}{\partial z}\right) & =-\frac{\partial p}{\partial z}+\eta\left[\frac{\partial u_{z}^{2}}{\partial x^{2}}+\frac{\partial u_{z}^{2}}{\partial z^{2}}\right] .
\end{aligned}
$$

Equations (9.6) to (9.8) are made dimensionless using the following scaling

$$
\begin{aligned}
& x^{*}=\frac{x}{L}, \quad z^{*}=\frac{z}{\alpha I}, \quad t^{*}=\frac{t V}{L}, \quad h^{*}=\frac{h}{\alpha I}, \\
& u_{x}^{*}=\frac{u_{x}}{V}, \quad u_{z}^{*}=\frac{u_{z}}{\alpha V}, \text { and } p^{*}=\frac{p}{V},
\end{aligned}
$$

where $\alpha$ is a small parameter of the same order as the channel slope. The lubrication

equation holds in geometries where $a \ll 1$. Upon substitution, the momentum equations yield (with asterisks suppressed hereafter) [4]:

$$
\begin{aligned}
\alpha \operatorname{Re}\left(\frac{\partial u_{x}}{\partial t}+u_{x} \frac{\partial u_{x}}{\partial x}+u_{z} \frac{\partial u_{x}}{\partial z}\right) & =-\frac{\partial p}{\partial x}+\alpha^{2} \frac{\partial^{2} u_{x}}{\partial x^{2}}+\frac{\partial^{2} u_{x}}{\partial z^{2}}, \\
\alpha^{3} \operatorname{Re}\left(\frac{\partial u_{z}}{\partial t}+u_{x} \frac{\partial u_{z}}{\partial x}+u_{z} \frac{\partial u_{z}}{\partial z}\right) & =-\frac{\partial p}{\partial z}+\alpha^{3} \frac{\partial^{2} u_{z}}{\partial x^{2}}+\alpha \frac{\partial^{2} u_{z}}{\partial z^{2}} .
\end{aligned}
$$

Since all dimensionless derivative terms in these two equations are of comparable order, the resulting dimensionless lubrication equations, in the limit of $\mathrm{a} \approx 0$ or $\mathrm{aRe} \approx 0$, are

$$
\begin{gathered}
-\frac{\partial p}{\partial x}+\frac{\partial u_{x}^{2}}{\partial z^{2}}=0 \\
-\frac{\partial p}{\partial z}=0
\end{gathered}
$$
and 
These equations are similar to those derived intuitively from channel flow, i.e., Eqs. (9.2) and (9.3). Notice that high Reynolds numbers are allowed as far as the product $\alpha$ Re is vanishingly small, and the flow remains laminar. The appropriate boundary conditions are:

(i) at $z=0, u_{x}=V$ (no-slip boundary condition);

(ii) at $z=h, u_{x}=0$ (slit flow, no slip boundary condition), or

(iii) at $z=h, \tau_{z x}=0$ (thin film, zero shear stress at free surface)

Under these conditions, the solution to Eq. (9.5) is

$$
\begin{array}{cc}
u_{x}=-\frac{1}{2 \eta} \frac{d p}{d x}\left(z h-z^{2}\right)+V\left(1-\frac{z}{h}\right) & \text { (slit slow), } \\
u_{x}=-\frac{1}{2 \eta} \frac{d p}{d x}\left(2 z h-z^{2}\right)+V . & \text { (film flow). }
\end{array}
$$

volume flux, and the pressure distribution in the lubricant layer can be calculated when the total flow rate, Q,

and the inclination, $\alpha$, are known. A lubrication layer will generate a positive pressure, and, hence, load capacity, normal to the layer only when the layer is arranged so that the relative motion of the two surfaces tends to drag fluid by viscous stresses from the wider to the narrower end of the layer. The load, W, supported by the pressure in slit flow is [5]

$$
W=\int_{0}^{L}\left(p-p_{0}\right) d x=\frac{6 \eta V}{\alpha^{2}}\left[\ln \left(\frac{d}{d-\alpha L}\right)-2\left(\frac{\alpha L}{2 d-\alpha L}\right)\right],
$$

where $\mathrm{d}$ is the height of the wide side of the converging channel, and $\mathrm{L}$ is the length of the channel. By decelerating the flow and by transmitting momentum, and thus load capacity to the boundary, the slope $\alpha$ is ultimately responsible for the pressure built-up.

9.1.3Reynolds Equation for Lubrication: Mass conservation on an infinitesimal volume yields

$$
Q_{x}-Q_{x+d x}=d x \frac{d h}{d t},
$$

which states that the convection of mass in the control volume is used to increase the fluid volume at a rate of

$$
\frac{d}{d t}(d x d h) \text {, }
$$

where $\mathrm{dx}$ and dh are respectively the width in the flow direction, and the height of the volume. By rearranging,

$$
-\frac{d Q}{d x}=\frac{d h}{d t}
$$

which, for confined and film flows, reduces respectively to

$$
\begin{aligned}
\frac{d}{d x}\left(-\frac{1}{2 \eta} \frac{d p}{d x} \frac{h^{3}}{6}+\frac{h V}{2}\right) & =-\frac{d h}{d t}, \\
\frac{d}{d x}\left(-\frac{1}{\eta} \frac{d p}{d x} \frac{h^{3}}{3}+h V\right) & =-\frac{d h}{d t} .
\end{aligned}
$$

Equations (9.18) and (9.19) represent the transient lubrication equations. The steady-state form of Eq. (9.18), 


$$
\begin{aligned}
& \frac{d}{d x}\left(-\frac{1}{2 \eta} \frac{d p}{d x} \frac{h^{3}}{6}+\frac{h V}{2}\right)=0, \\
& -\frac{1}{2 \eta} \frac{d p}{d x} \frac{h^{3}}{6}+\frac{h V}{2}=Q, \quad \text { calculated by }
\end{aligned}
$$

is integrated to

and the pressure is

$$
\begin{aligned}
& p(x)=p_{0}+6 \eta V \int_{0}^{x} \frac{d x}{h^{2}(x)}-12 \eta Q \int_{0}^{x} \frac{d x}{h^{3}(x)}, \\
& Q=\frac{\left(p_{0}-p_{L}\right)}{12 \eta \int_{0}^{L} h^{-3}(x) d x}+\frac{V}{2} \frac{\int_{0}^{L} h^{-2}(x) d x}{\int_{0}^{L} h^{-3}(x) d x} .
\end{aligned}
$$$$
\text { is }
$$

where

$$
W=\int_{0}^{L}\left|p_{0}-p(x)\right| d x,
$$

The load capacity

friction on the same surface is

$$
F=-\int_{0}^{L} \tau_{z x} d x
$$

It is easy to show that the load capacity is of order $\alpha-2$, whereas the shear or friction is of order $\alpha-1$. Thus, the ratio load/friction increases with $\alpha-1$. Important applications of the lubrication theory for confined flows are journalbearing [2,6], and piston-ring lubricated systems of engines [7]. Other flows that can be studied by means of the lubrication equations include wire coating [8], roll coating [9], and many polymer applications [10]. Starting from Eq. (9.17), the solution to these problems follows the procedure outlined above. The flow rate is often given by

$$
Q=V h_{f}
$$

where $\mathrm{V}$ is the speed of production and hf is the final target thickness. The boundary condition on the pressure at the outlet may vary:

$$
p(L)=0, d p(L) / d x=0, p(L)=f_{\sigma},_{\text {(sthere to i i the }}
$$

force per unit area due to surface tension) and combinations of them [11]. In confined lubrication flows, pressure build-up develops due to inclination, $\alpha$, that may result in backflow of some of the entering liquid. This pressure is used to support loads. In typical thin-film lubrication flows, any pressure build-up is primarily due to surface tension. In fact, if surface tension is negligible, then the pressure gradient is zero. For film lubrication flows, the steady-state form of Eq. (9.19),

is integrated to

$$
\frac{d}{d x}\left(-\frac{1}{\eta} \frac{d p}{d x} \frac{h^{3}}{3}+V h\right)=0
$$




$$
-\frac{1}{\eta} \frac{d p}{d x} \frac{h^{3}}{3}+V h=Q=V h_{f},
$$

where the film thickness, $\mathrm{h}$, is unknown. However, the pressure gradient, dp/dx, can be deduced from surface tension, by means of the Young-Laplace [12] equation. By using the lubrication assumption that the slope, $\mathrm{dh} / \mathrm{dx}$, must be much smaller than unity, we get

$$
-p=\frac{\sigma \frac{d^{2} h}{d x^{2}}}{\left[1+\left(\frac{d h}{d x}\right)^{2}\right]^{1 / 2}} \simeq \sigma \frac{d^{2} h}{d x^{2}} .
$$

Here $h(x)$ is the elevation of the free surface from the $x$-axis, and $\sigma$ is the surface tension of the liquid. Then,

$$
-\frac{d p}{d x}=\sigma \frac{d^{3} h}{d x^{3}} \text {. }
$$

Substituting Eq. (9.29) in Eq. (9.27) we get

$$
\frac{\sigma}{3 \eta} h^{3} \frac{d^{3} h}{d x^{3}}+h V=V h_{f}
$$

which is rearranged to

$$
h^{3} \frac{d^{3} h}{d x^{3}}+3 C a\left(h-h_{f}\right)=0 .
$$

Equation (9.31) is nonlinear and cannot be solved analytically.

Some important applications of the thin-film lubrication equations are films falling under surface tension [11], dip and extrusion coating [6], and wetting and liquid spreading [12]. A similar class of problems includes centrifugal spreading which is common in bell sprayers and in spin coating [3, 12]. A rich collection of lubrication problems from polymer processing can be found in the relevant literature [13, 14], and from recent work on coating $[15,16]$.

\section{Conclusion-}

According to all the theoretical and experimental evidence we can consider some parameter which can explain the instability of streaming fluid in porous Rivlin-Ericksen medium.Among them mobility ratio is a important parameter which is used primarily in flows through porous media, where steam is often injected to displace the more viscous oil $[12,23]$. Intuitively, highly viscous and elastic materials can be stretched the most. This poses significant experimental challenges in producing ideal, extensional flows in order to measure elongational viscosity of viscoelastic liquids of low shear viscosity. This need does not exist in Newtonian liquids, the elongational viscosity of which is exactly three times the shear viscosity, by virtue of Newton's law of viscosity. At this stage, fiber-spinning and other related operations (e.g., falling curtains and fibers under gravity) and the recent opposing jet method [24] appear to provide the best means (though not perfect [25]), to measure elongational viscosity. The elongational viscosity is extremely important in industrial polymer processes which may involve any kind of extensional deformation, given that

(a) the common shear viscosity measurements do not provide any indication of the magnitude of the elongational viscosity at even moderate stretching or compression, and

(b) the elongational viscosity may attain values ten-fold or even higher than the shear viscosity, which gives rise to huge normal stresses and therefore, to excessively high drawing forces and compressive loads, required to process highly elastic viscoelastic polymer melts or solutions.

\section{Primary Studies-}

\section{References:-}

\section{SET-I:}

[1]. Alfven, H. and Carlquist, P., “The Problem of Dust and Suspended Particles in Gas Particle Medium”, Astrophys. Space. Sc., Vol. 55, (1978), 487.

[2]. Scanlon, J.H. and Segel, L.A., "The Problems of Suspended Particles on the Onset of B'enard Convection”, Phys. Fluids, Vol. 17, (1973), 1573.

[3]. Sharma, R.C., Prakash, K. and Dube, S.N., "Effect of Suspended Particles on the Onset of B'enard Problem in Hydromagnetics", Acta Physica Hungarica, Vol. 40, No. 2, (1976), 680. 
[4]. Sharma, R.C., "Effect of Magnetic Field on the Gravitational Instability of Self Gravitating Homogeneous Gas Particle Medium in the Presence of Suspended Particles", Astrophys. Space Sc., Vol. 46, (1977), 255.

[5]. Chhajlani, R.K. and Sanghvi, R.K., "Magneto-Gravitational Instability of a Fluid Through Porous Medium Including Finite Ion Larmor Radius", Contrib. Plasma Phys., Vol. 25, No. 6, (1985), 623.

[6]. Sunil Sharma, R.C. and Chandel, R.S., "Stability of Stratified Rivlin-Ericksen Fluid-Particle Mixture in Hydromagnetics in Porous Medium", Ganita, Vol. 51, No. 2, (2000), 179.

[7]. Sharma, R.C. and Kumar, P., "Effect of Suspended Particles on Thermal Instability in Rivlin-Ericksen Elastico-Visocus Medium", Indian J. Pure Appl. Maths., Vol. 30, No. 5, (1999), 477.

[8]. Chandrasekhar, S., "Hydrodynamic and Hydromagnetic Stability", Dover Publications, New York, U.S.A., (1981).

[9]. Kumar, P., "Rayleigh-Taylor Instability of Rivlin-Ericksen Elastico-Viscous Fluids in Presence of Suspended Particles Through Porous Medium", India J. Pure Appl. Math., Vol. 31, No. 5, (2000), 533.

[10]. Bhatia, P.K., "Rayleigh-Taylor Instability of Two Viscous Superposed Conducting Fluids in the Presence of a Uniform Horizontal Magnetic Field", Nuov. Cim., Vol. 19B, (1974), 161.

[11]. Sharma, R.C. and Thakur, K.D., "Stability of Superposed Fluids in the Presence of a Variable Magnetic Field", Czech J. Phys., Vol. 50, (2000), 753 .

[12]. Rayleigh, L., "The Equilibrium of an Incompressible, Non-Viscous Fluid of Variable Density Stratified in the Vertical Direction", Proc. Lond. Soc., Vol. 14, (1983), 170.

[13]. Sharma, V. and Rana, G.C., "Thermosolutal Instability of Rivlin-Ericksen Rotating Fluid in the Presence of Magnetic Field and Variable Gravity Field in Porous Medium”, Proc. Nat. Acad. Sci. India, Vol. 73, No. A, (2003), 1.

[14]. Pundir, S.K. and Bahadur, R., "Stability of Stratified Visco-Elastic Walter's (Model B') Dusty Fluid in Porous Medium", Acta Ciencia Indica, Vol. 3, 703 (2005), 703-709.

[15]. Kumar, P., Mohan, H. and Lal, M., "The Effect of Magnetic Field on Thermal Instability of Rotating Rivlin-Ericksen Visco-Elastic Fluid", Int. J. of Maths. and Mathematics Sc., Vol. 28042, (2006), 1.

\section{SET-II:}

[1] CHANDRASEKHAR S., Hydrodynamic and Hydromagnetic Stability, Dover Publications, New York, 1981.

[2] LAPWOOD E.R., Convection of a fluid in a porous medium, Proc. Camb. Phil. Soc., 1948, 44, 508.

[3] WOODING R.A., Rayleigh instability of a thermal boundary layer in flow through a porous medium, J. Fluid Mech., $1960,9,183$.

[4] SHARMA R.C., KUMAR P., Thermal instability of an Oldroydian viscoelastic fluid in porous medium, Engng. Trans, Poland, 1996, $14,99$.

[5] RIVLIN R.S., ERICKSEN J.L., Stress-deformation relations for isotropic material, J. Rational Mech. Anal., $1955,4,323$.

[6] STOMMEL H., FEDOROR K.N., Small scale structure in temperature and salinity near Timor and Mindanao, Tellus, $1967,19,306$.

[7] LINDEN P.F., Salt fingers in a steady shear flow, Geophys. Fluid Dynamics, 1974, 6, 1.

[8] LISTER C.R.B., On the thermal balance of a midocean ridge, Geophys. J. Roy. Astr. Soc., 1972, $26,515$.

[9] McDONNEL J.A.M., Cosmic Dust, John Wiley and Sons, Toronto, 1978.

[10] PRADHAN G.K., SAMAL P.C., Thermal instability of fluid layer under variable body forces, J. Math. Anal. Appl., $1987,122,487$.

[11] SHARMA V., RANA G.C., Thermal instability of a Walters' (Model B') elastico-viscous fluid in the presence of variable gravity field and rotation in porous medium, J. Non-Equilib. Thermodyn., 2001, $26,31$.

\section{SET-III:}

[1]. Allah, M.H.O., "Rayleigh-Taylor Stability in the Presence of Rotation", Astrophysics and Space Science, 175, 149-155, 1991.

[2]. Allah, M.H.O., "Rayleigh-Taylor Instability with Surface Tension, Porous Media, Rigid Planes and Exponential Densities", Indian J. Pure and Applied Maths, 33, 1391-1404, 2002.

[3]. Benjamin, T.B. and Bridges, T.J., "Reappraisal of the Kelvin-Helmholtz Problem Part 1, Hamiltonian Structure", J. Fluid Mech., 333, 301-325, 1997.

[4]. Bhatia, P.K., "Rayleigh-Taylor Instability of Two Viscous Superposed Conducting Fluids", Nuovo Cimento, 19, $161-168,1974$.

[5]. Bhatia, P.K. and Mathur, R.P., "Stability of Viscous Rotating Gravitating Streams in a Magnetic Field", Z. Naturforsch., 61, 258-262, 2006.

\section{Secondary Studies :- \\ SET-I:}

[1] Chandrasekhar S., Hydrodynamic and Hydromagnetic Stability, Dover Publication, New York, 1981.

[2] Francis J.R.D., Phil. Mag. Ser.7, 45(1954), 695.

[3] Johri A.K., Acta Ciencia Indica, 24(1976), 377.

[4] Sisodia S.S. and Gupta M., Ind. Jour. Theor. Phys., 32(1984), 5.

[5] Srivastava R.K. and Singh K.K., Bull. Cal. Math. Soc., 80(1988), 286.

[6] Sharma R.C. and Kumar P.Z., Naturforsch, 51a(1996), 881.

[7] Phillips O.M., Flow and Reaction in Permeable Rocks, Cambridge University Press, Cambridge, 1991.

[8] McDonnel J.A.M., Cosmic Dust, John Wiley and Sons, Toronto, 1978, p.330.

[9] Sharma R.C. and Spanos T.J.T., Canadian J. Phys., 60(1982), 1391.

\section{SET-II:}

[1]. S. O. Ajadi, A note on the unsteady flow of dusty-viscous fluid between two parallel plates, J. Appl. Math. and Computing 18(2) (2005), 393-403.

[2]. P. K. Bhatia and J. M. Steiner, Convective instability in a rotating viscoelastic fluid layer, Z. Angew. Math. Mech. 52 (1972), $321-$ 327.

[3]. S. Chandrasekhar, Hydrodynamic and Hydromagnetic Stability, Dover Publication, New York, 1981.

[4]. J. E. Dunn and K. R. Rajagopal, Fluids of differential type: Critical review and thermodynamic analysis, Int. J. Engg. Sci. 33(5) (1995), 689-729.

[5]. A. S. Gupta, Hall effects on thermal instability, Rev. Roum. Math. Pures Appl. 12 (1967), 665-677.

[6]. U. Gupta, Proceedings of 50th congress of ISTAM (an international meet), IIT Kharagpur (2005), 133-141. 